\title{
Colossal magnetoresistance in a nonsymmorphic antiferromagnetic insulator
}

Priscila Rosa $\mathbb{i D}^{1 凶}$, Yuanfeng $\mathrm{Xu}^{2}$, Marein Rahn ${ }^{1}$, Jean Souza ${ }^{3}$, Satya Kushwaha ${ }^{1,4}$, Larissa Veiga ${ }^{5}$, Alessandro Bombardi ${ }^{6}{ }^{6}$, Sean Thomas ${ }^{1}$, Marc Janoschek ${ }^{7}$, Eric Bauer (D) ${ }^{1}$, Mun Chan ${ }^{4}{ }^{4}$, Zhijun Wang ${ }^{8,9}$, Joe Thompson ${ }^{1}$, Neil Harrison ${ }^{4}$, Pascoal Pagliuso ${ }^{3}$, Andrei Bernevig ${ }^{10}$ and Filip Ronning ${ }^{1}$

Here we investigate antiferromagnetic $\mathrm{Eu}_{5} \mathrm{In}_{2} \mathrm{Sb}_{6}$, a nonsymmorphic Zintl phase. Our electrical transport data show that Eu $\mathrm{In}_{2} \mathrm{Sb}_{6}$ is remarkably insulating and exhibits an exceptionally large negative magnetoresistance, which is consistent with the presence of magnetic polarons. From ab initio calculations, the paramagnetic state of $E_{5} \ln _{2} \mathrm{Sb}_{6}$ is a topologically nontrivial semimetal within the generalized gradient approximation (GGA), whereas an insulating state with trivial topological indices is obtained using a modified Becke-Johnson potential. Notably, GGA $+U$ calculations suggest that the antiferromagnetic phase of Eu $\mathrm{In}_{2} \mathrm{Sb}_{6} \mathrm{may}_{\mathrm{g}} \mathrm{host}$ an axion insulating state. Our results provide important feedback for theories of topological classification and highlight the potential of realizing clean magnetic narrow-gap semiconductors in Zintl materials.

npj Quantum Materials (2020)5:52 ; https://doi.org/10.1038/s41535-020-00256-8

\section{INTRODUCTION}

Narrow-gap semiconductors exhibit a breadth of striking functionalities ranging from thermoelectricity to dark matter detection $^{1,2}$. More recently, the concept of topological insulating phases in bulk materials has renewed the interest in this class of materials $s^{3-5}$. Independent of the target application, a primary goal from the experimental point of view is the synthesis of genuine insulators free of self doping. Materials design is usually guided by simple electron count (e.g. tetradymite $\mathrm{Bi}_{2} \mathrm{Te}_{3}{ }^{6}$ ), correlated gaps (e.g. Kondo insulators $\mathrm{SmB}_{6}{ }^{7}$ and $\mathrm{YbB}_{12}{ }^{8}$ ) or the Zintl concept (e.g. $\mathrm{Sr}_{2} \mathrm{~Pb}^{9}$ and $\left.\mathrm{BaCaPb}^{10}\right)$. Zintl phases are valence precise intermetallic phases formed by cations (alkaline, alkaline-earth and rare-earth elements) and covalently bonded (poly)anionic structures containing post-transition metals. The electron transfer between these two entities gives rise to an insulating state, whereas the inclusion of rare-earth elements allows for magnetism, which breaks time-reversal symmetry and may promote new quantum ground states ${ }^{11-13}$

The myriad of crystal structures within the Zintl concept provides a promising avenue to search for clean semiconductors. Here we experimentally investigate Zintl $\mathrm{Eu}_{5} \mathrm{In}_{2} \mathrm{Sb}_{6}$ in single crystalline form. Low-carrier density magnetic materials containing Europium are prone to exhibiting colossal magnetoresistance $(C M R)^{14-18}$. The strong exchange coupling between the spin of the carriers and the spins of the $\mathrm{Eu}^{2+}$ background causes free carriers at low densities to self-trap in ferromagnetic clusters around the Eu sites, which gives rise to a quasiparticle called magnetic polaron ${ }^{19}$. This quasiparticle has been identified in several Zintl materials ranging from simple cubic $\mathrm{EuB}_{6}{ }^{17,20}$ to monoclinic $\mathrm{Eu}_{11} \mathrm{Zn}_{4} \mathrm{Sn}_{2} \mathrm{As}_{12}{ }^{18}$. Most CMR compounds have a ferromagnetic ground state, including doped magnanites $\mathrm{RE}_{1-x} A_{x} \mathrm{MnO}_{3}$ (RE $=$ rare-earth, $A=$ divalent cation) in which CMR was first observed $^{21,22}$. EuTe and $\mathrm{Eu}_{14} \mathrm{MnBi}_{11}$, however, revealed the possibility of realizing CMR in antiferromagnets ${ }^{14,16,23}$, which also brings promise for applications due to their small stray fields ${ }^{24}$.

Additionally, nonsymmorphic symmetries are expected to be particularly powerful in creating protected band crossings and surface states, which provide an additional organizing principle within the Zintl concept ${ }^{25,26}$. For instance, Wieder et al. predicted that Zintl $\mathrm{Ba}_{5} \mathrm{In}_{2} \mathrm{Sb}_{6}$, the non- $f$ analog of $\mathrm{Eu}_{5} \mathrm{In}_{2} \mathrm{Sb}_{6}$, hosts fourfold Dirac fermions at $\bar{M}$ connected to an hourglass fermion along $\overline{\Gamma X}^{27}$. Recent attempts to theoretically catalog all known uncorrelated materials indicate that $\mathrm{Ba}_{5} \mathrm{In}_{2} \mathrm{Sb}_{6}$ may be classified as a topological insulator ${ }^{28,29}$ or trivial insulator ${ }^{30,31}$. This discrepancy begs for an experimental investigation.

$\mathrm{Eu}_{5} \mathrm{In}_{2} \mathrm{Sb}_{6}$, just like its $\mathrm{Ba}$ analog, crystallizes in space group Pbam. As expected from the $4 f$ localized moments in multiple sites, $\mathrm{Eu}_{5} \mathrm{In}_{2} \mathrm{Sb}_{6}$ orders antiferromagnetically at $T_{\mathrm{N} 1}=14 \mathrm{~K}$ in a complex magnetic structure. Remarkably, CMR sets in at $15 T_{\mathrm{N} 1}$ and is accompanied by an anomalous Hall component. Our data collectively point to the presence of magnetic polarons. To shed light on the topology of the band structure of $\mathrm{Eu}_{5} \mathrm{In}_{2} \mathrm{Sb}_{6}$, we have performed first-principles calculations using different functionals and magnetic phases. Though an insulating state with trivial topological indices is obtained using modified Becke-Johnson (mBJ) functional in the paramagnetic state, topological nontrivial states with strong indices emerge in the generalized gradient approximation (GGA) + U calculations within putative antiferromagnetic states.

\section{RESULTS}

Magnetic susceptibility measurements

We first discuss the thermodynamic properties of $\mathrm{Eu}_{5} \mathrm{In}_{2} \mathrm{Sb}_{6}$ single crystals. Figure 1a highlights the complex anisotropy in the

\footnotetext{
${ }^{1}$ Quantum Group, Los Alamos National Laboratory, Los Alamos, NM 87545, USA. ${ }^{2}$ Max Planck Institute of Microstructure Physics, 06120 Halle, Germany. ${ }^{3}$ Instituto de Física "Gleb Wataghin", UNICAMP, Campinas - SP 13083-859, Brazil. "National High Magnetic Field Laboratory, Los Alamos National Laboratory, MS E536, Los Alamos, NM 87545, USA ${ }^{5}$ Department of Physics and Astronomy, University College London, Gower Street, London WC1E 6BT, UK. ${ }^{6}$ Diamond Light Source, Harwell Science and Innovation Campus, Didcot OX11 ODE, UK. ' Laboratory for Neutron and Muon Instrumentation, Paul Scherrer Institut, Villigen, Switzerland. ${ }^{8}$ Beijing National Laboratory for Condensed Matter Physics, and Institute of Physics, Chinese Academy of Sciences, 100190 Beijing, China. ${ }^{9}$ University of Chinese Academy of Sciences, 100049 Beijing, China. ${ }^{10}$ Department of Physics, Princeton University, Princeton, NJ 08544, USA. ${ }^{凶}$ email: pfsrosa@lanl.gov
} 

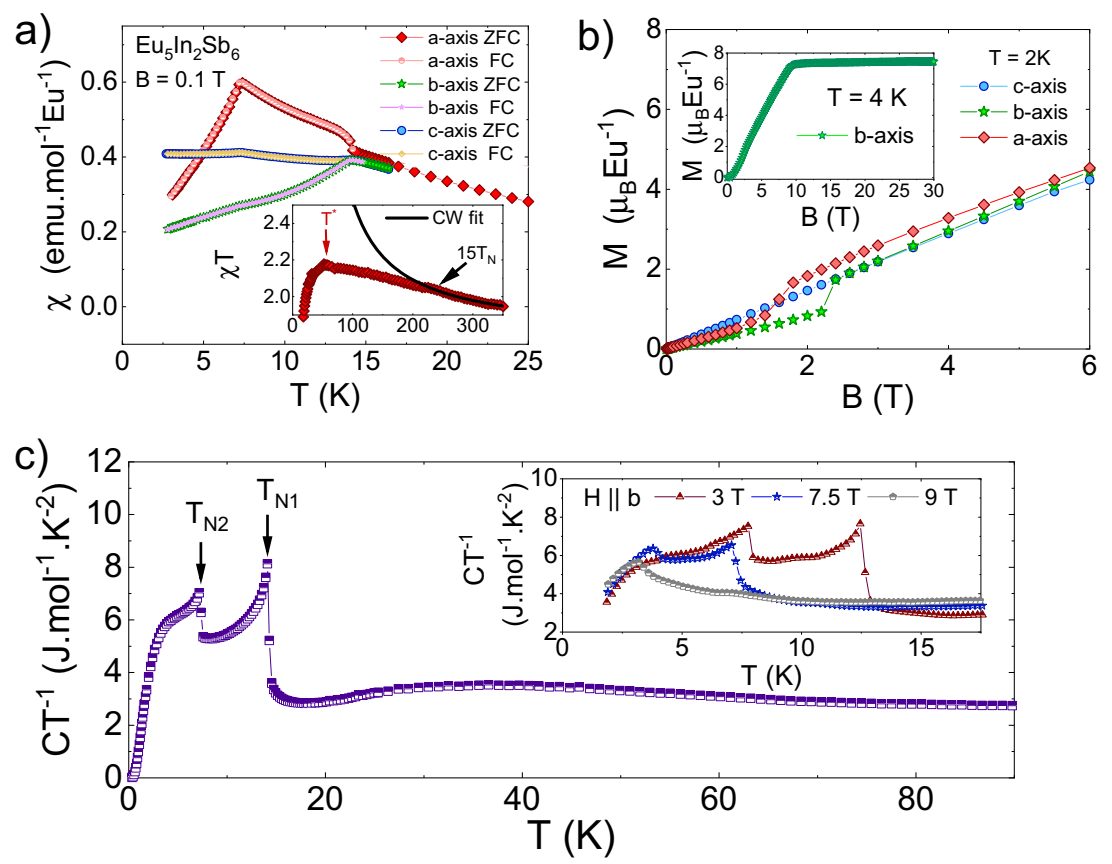

Fig. 1 Thermodynamic properties of $\mathrm{Eu}_{\mathbf{5}} \mathbf{I n}_{\mathbf{2}} \mathrm{Sb}_{\mathbf{6}}$ crystals. a Magnetic susceptibility, $X(T)$, in both zero-field-cooled (ZFC) and field-cooled (FC) sweeps. Inset shows XT. Black solid line shows the high-temperature CW fit. b Magnetization vs applied field at $2 \mathrm{~K}$. Inset shows high-field magnetization data at $4 \mathrm{~K}$. c Zero-field specific heat as a function of temperature. Inset shows $C / T$ at different applied fields.

low-temperature magnetic susceptibility of $\mathrm{Eu}_{5} \mathrm{In}_{2} \mathrm{Sb}_{6}$. Two magnetic transitions can be identified at $T_{\mathrm{N} 1}=14 \mathrm{~K}$ and $T_{\mathrm{N} 2}=7 \mathrm{~K}$, in agreement with previous measurements on polycrystalline samples ${ }^{32}$. One can also infer that the $c$-axis is the magnetization hard-axis and that the moments lie in the $a b$-plane. No hysteresis is observed between zero-field-cooled and field-cooled measurements at $0.1 \mathrm{~T}$, which rules out hard ferromagnetic order or spinglass behavior; however, a small in-plane ferromagnetic component $\left(0.06 \mu_{B}\right)$ is observed at very low fields $(B \leq 0.1 \mathrm{~T})$, indicative of a complex magnetic structure with canted moments (see Supplementary Fig. 1).

The inset of Fig. 1a shows the product of magnetic susceptibility and temperature as a function of temperature. At high temperatures $(T>225 \mathrm{~K})$, a Curie-Weiss $(\mathrm{CW})$ fit yields a ferromagnetic (FM) Weiss temperature of $\theta=30 \mathrm{~K}$ despite the antiferromagnetic (AFM) order at low temperatures, which further corroborates the presence of a complex magnetic configuration with multiple exchange interactions. The inverse of the magnetic susceptibility is shown in Supplementary Fig. 3. The CW fit also yields an effective moment of $8 \mu_{\mathrm{B}} \mathrm{Eu}^{-1}$, in good agreement with the Hund's rule moment of $7.94 \mu_{\mathrm{B}} \mathrm{Eu}^{-1}$ for $\mathrm{Eu}^{2+}$. In fact, our X-ray absorption spectra at the Eu $L$ edges ${ }^{33}$ confirm that all three Eu sites are divalent (see Supplementary Fig. 5). Previous X-ray absorption studies observed a finite $\mathrm{Eu}^{3+}$ component, which could be due to an impurity phase present in polycrystalline samples ${ }^{32}$. The fully divalent character of europium in $\mathrm{Eu}_{5} \mathrm{In}_{2} \mathrm{Sb}_{6}$ has been recently confirmed by Mossbauer measurements ${ }^{34}$.

Notably, our magnetic susceptibility data deviate from the CW fit at temperatures well above the ordering temperature (inset of Fig. 1a). In purely divalent compounds such as $\mathrm{Eu}_{5} \mathrm{In}_{2} \mathrm{Sb}_{6}, \mathrm{Eu}^{2+}$ is a localized $S$-only ion $(J=S=7 / 2)$, which implies crystal-field and Kondo effects to be negligible to first order. As a result, the deviation from a CW fit indicates the presence of short-range magnetic interactions as observed previously in the manganites $\mathrm{RE}_{1-x} A_{x} \mathrm{MnO}_{3} \quad(\mathrm{RE}=$ rare-earth, $A=$ divalent cation). Based on small-angle neutron scattering measurements, this deviation was argued to be due to the formation of magnetic polarons ${ }^{35}$. As temperature decreases, magnetic polarons are expected to grow in size and eventually overlap when $n \xi^{3} \approx 1$, where $n$ is the carrier density and $\xi$ is the magnetic correlation length ${ }^{36}$. The inset of Fig. 1a shows a sharp decrease in $X(T) T$ at $T^{*} \sim 40 \mathrm{~K}$, which reflects the onset of strong antiferromagnetic correlations between polarons. Figure $1 \mathrm{~b}$ shows the low-temperature anisotropic magnetization of $\mathrm{Eu}_{5} \mathrm{In}_{2} \mathrm{Sb}_{6}$. The hard c-axis magnetization increases linearly with field, whereas a field-induced transition is observed within the basal plane before saturation is reached at about $10 \mathrm{~T}$ (inset of Fig. 1b). Figure 1c shows the temperature dependence of the specific heat, $C$, at zero field. In agreement with magnetic susceptibility data, $C / T$ exhibits two phase transitions at $T_{\mathrm{N} 1}$ and $T_{\mathrm{N} 2}$ as well as a magnon contribution below $T_{N 2}$, typical of $\mathrm{Eu}^{2+}$ compounds. The entropy recovered at $T_{\mathrm{N} 1}$ is about $90 \%$ of $R \ln 8$ (not shown), the expected entropy from the $\operatorname{Eu}^{2+}(J=7 / 2)$ ground state. The extrapolation of the zero-field $C / T$ to $T=0$ gives a Sommerfeld coefficient of zero within the experimental error, indicating that $\mathrm{Eu}_{5} \mathrm{In}_{2} \mathrm{Sb}_{6}$ is an insulator with very small amounts of impurities. A Schottky-like anomaly at about $35 \mathrm{~K}$ indicates the presence of short-range correlations, in agreement with magnetic susceptibility data at $T^{*}$. The inset of Fig. 1c displays the field dependence of the low-temperature transitions when field is applied along the $b$-axis. The transitions are mostly suppressed by $9 \mathrm{~T}$, in agreement with the saturation in magnetization.

\section{Electrical transport measurements}

We now turn our attention to electrical transport data. Figure $2 a$ shows the temperature-dependent electrical resistivity, $\rho(T)$, of $\mathrm{Eu}_{5} \mathrm{In}_{2} \mathrm{Sb}_{6}$ measured with current along the $c$-axis. Remarkably, $\rho$ $(T)$ rises by almost six orders of magnitude in the paramagnetic state, in agreement with the clean insulating response observed in $C / T$ but in stark contrast to $\rho(T)$ measurements in polycrystals ${ }^{37}$. Below $T_{\mathrm{N} 1}, \rho(T)$ decreases by three orders of magnitude, pointing to the overlap of magnetic polarons within the antiferromagnetic state. Finally, at lower temperatures $\rho(T)$ rises again, and a small kink is observed at $T_{\mathrm{N} 2}$.

The high-temperature electrical resistivity can be fit to an activated behavior given by $\rho_{0} \tilde{T}^{n} \exp \left(E_{\mathrm{a}} / k_{\mathrm{B}} T\right)$ (inset of Fig. 2a), 

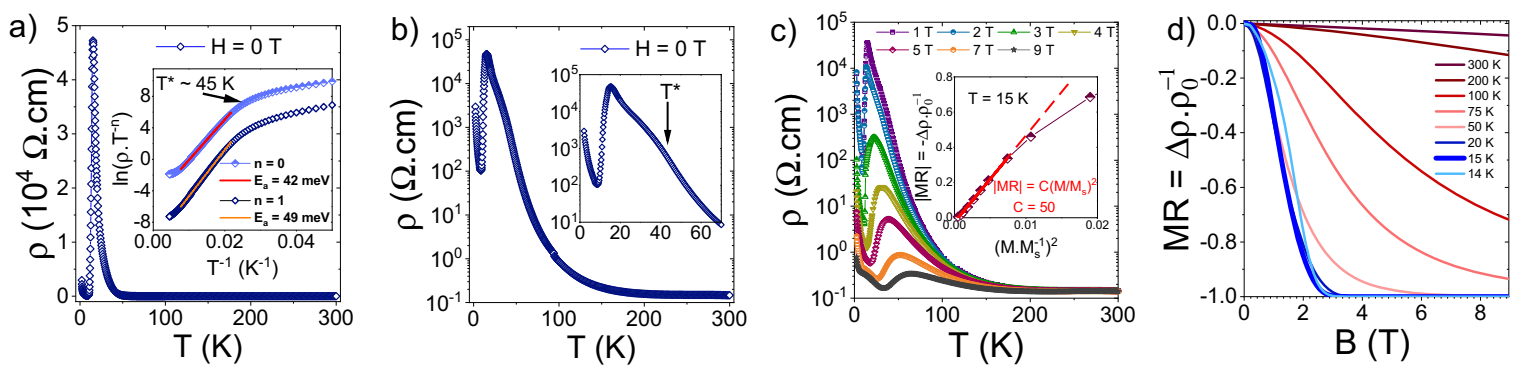

Fig. 2 Electrical resistivity measurements on $\mathbf{E u}_{5} \mathbf{I n}_{\mathbf{2}} \mathbf{S b}_{\mathbf{6}}$ single crystals. a Temperature-dependent electrical resistivity, $\rho(T)$, at zero field. Electrical current was applied along the $c$-axis. Inset shows an activated log $\rho$ vs $1 / T$ plot. $\mathbf{b} \rho(T)$ in a log plot. Inset shows a zoom in the lowtemperature region. $\mathbf{c} \rho(T)$ at various magnetic fields applied long the $b$-axis. Inset shows MR vs reduced magnetization $\left(M / M_{\mathrm{s}}\right) s q u a r e d$. $\mathbf{d} M R$ vs applied field at various temperatures.

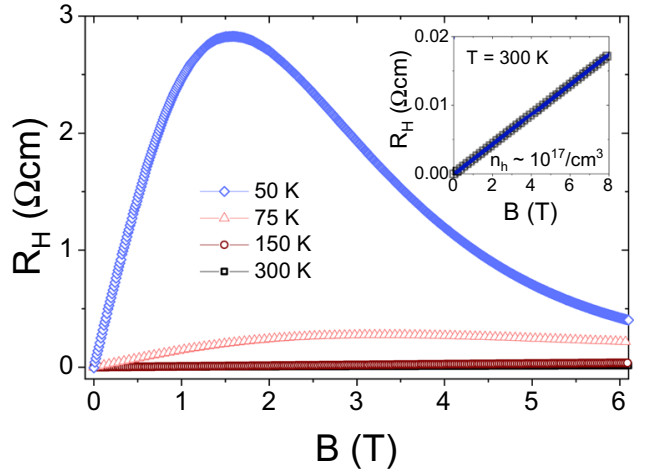

Fig. 3 Hall effect of $\mathrm{Eu}_{5} \mathbf{I n}_{\mathbf{2}} \mathrm{Sb}_{6}$ single crystals. Hall resistivity vs applied field at various temperatures. Current is applied along the $c$ axis and fields are along the $b$-axis. Inset shows the linear Hall response at $300 \mathrm{~K}$.

where $\tilde{T}$ is the reduced temperature. For $n=0$, the Arrhenius plot yields a narrow gap of $40 \mathrm{meV}$ whereas a slightly larger energy is extracted when $n=1$ for adiabatic small-polaron hopping conduction $^{38}$. From these data alone, it is not possible to differentiate between the two mechanisms. Nevertheless, the activated behavior breaks down at about $T^{*} \sim 40 \mathrm{~K}$, indicating that another mechanism is present. This energy scale is more pronounced in a log plot shown in Fig. $2 b$.

The evolution of the CMR in $E_{5} I_{2} \mathrm{Sb}_{6}$ is summarized in Fig. $2 \mathrm{~d}$. Though the negative magnetoresistance is small at room temperature, it rapidly increases below about $15 T_{\mathrm{N} 1}$. At liquid nitrogen temperatures $(T \sim 75 \mathrm{~K})$, for instance, the $M R$ reaches $-50 \%$ at only $3 \mathrm{~T}$ and $-94 \%$ at $9 \mathrm{~T}$. Ultimately, the MR peaks at $-99.999 \%$ at $9 \mathrm{~T}$ and $15 \mathrm{~K}$. This is, to our knowledge, the largest CMR observed in a stoichiometric antiferromagnetic compound.

Hall measurements provide valuable information on the type of carriers and the scattering mechanisms in a material. Figure 3 shows the Hall resistivity, $R_{\mathrm{H}} \equiv \rho_{x z}$, for fields applied along the $b$-axis of $\mathrm{Eu}_{5} \mathrm{In}_{2} \mathrm{Sb}_{6}$. At room temperature, $R_{\mathrm{H}}$ is linear, as expected from a nonmagnetic single-band material (inset of Fig. 3a). The positive slope, $R_{0}$, implies positive (hole) carriers and a carrier density of $n_{\mathrm{h}}=1 / R_{0} e=10^{17} / \mathrm{cm}^{3}$, typical of narrow-gap semiconductors.

As the temperature is lowered, however, a nonlinear $R_{H}$ component sets in at about $15 T_{\mathrm{N} 1}$, the same temperature at which CMR emerges. As the band structure of this band insulator is not expected to change dramatically in this temperature range, our result may indicate that the formation of magnetic polarons is responsible for the anomalous Hall effect (AHE). We note, however, that the presence of multiple carriers cannot be ruled out at this time. Though the ferromagnetic nature of the magnetic

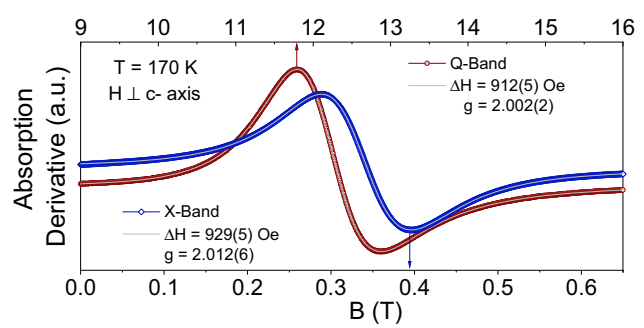

Fig. 4 Electron spin resonance of $\mathrm{Eu}_{5} \mathrm{In}_{2} \mathrm{Sb}_{6}$ single crystals. ESR spectra at $170 \mathrm{~K}$ for $X(f=9.5 \mathrm{GHz})$ and $Q(f=34 \mathrm{GHz})$ bands.

polaron cluster is a natural explanation for the anomalous contribution, a quantitative analysis of the various intrinsic and extrinsic contributions to the AHE will require determining the anisotropic conductivity tensor using micro-fabricated devices, including the region below $50 \mathrm{~K}$.

\section{Electron spin resonance measurements}

We complete our experimental investigation with microscopic electron spin resonance (ESR) measurements. Electron spin resonance is a site-specific spectroscopic technique, and $\mathrm{Eu}^{2+}$ ions are particularly suitable paramagnetic probes because of their S-only state ${ }^{39,40}$. The $\mathrm{Eu}^{2+}$ ESR spectra of $\mathrm{Eu}_{5} \mathrm{In}_{2} \mathrm{Sb}_{6}$ in the paramagnetic state, shown in Fig. 4, consists of a single unresolved resonance (i.e., no fine or hyperfine structure). The ESR linewidth, $\Delta H$, provides information on the interactions of the spins with their environment and their motion. In the case of semimetallic $\mathrm{EuB}_{6}$, the $\mathrm{Eu}^{2+} \Delta H$ was claimed to be dominated by spin-flip scattering due to the exchange between $4 f$ and conduction electrons ${ }^{39}$. As a result, $\Delta H$ narrows at higher fields due to a reduction in the spin-flip scattering, consistent with the presence of magnetic polarons. The linewidth of $\mathrm{Eu}_{5} \mathrm{In}_{2} \mathrm{Sb}_{6}$ also narrows at higher fields ( $Q$-band) when compared to low fields $\left(X\right.$-band), though not as strongly as in $\mathrm{EuB}_{6}{ }^{39}$. This narrowing further indicates that the resonance is homogeneous in the paramagnetic state. In the case of a small-gap insulator as $\mathrm{Eu}_{5} \mathrm{In}_{2} \mathrm{Sb}_{6}$, the $\mathrm{Eu}^{2+} \mathrm{ESR}$ linewidth is dominated by spin-spin interactions ${ }^{39,41,42}$. The resulting relaxation mechanism is set by $T_{2}$, the spin-spin relaxation time, which in turn is affected by the distribution of Eu-Eu exchange interactions and internal fields. An applied magnetic field causes an increase in $T_{2}$ as the size of the ferromagnetic polaron grows, which results in the observed ESR line narrowing. At the same time, the $g$-value decreases as a function of magnetic field, which indicates an antiferromagnetic inter-polaron coupling. Therefore, our ESR results are also consistent with the presence of magnetic polarons in $\mathrm{Eu}_{5} \mathrm{In}_{2} \mathrm{Sb}_{6}$. More detailed ESR measurements will be the focus of a separate study. 

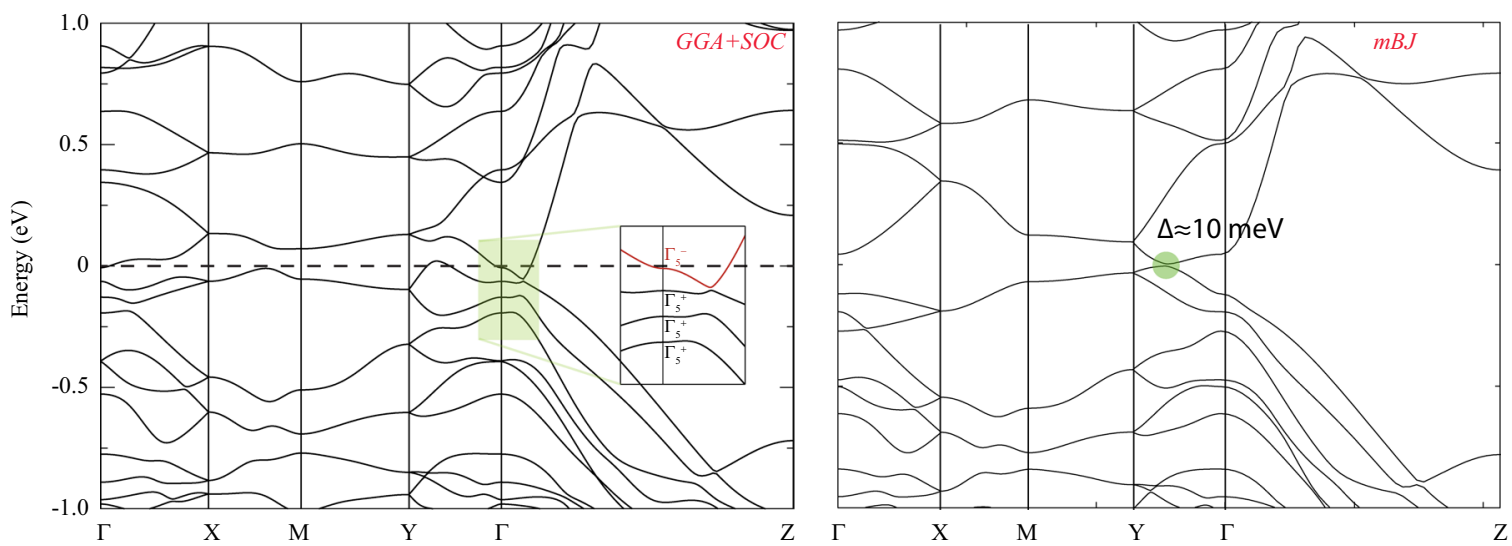

Fig. 5 Band structure calculations for $E_{u_{5}} \mathbf{I n}_{2} \mathbf{S b}_{6}$. Calculations in the paramagnetic state using the GGA + SOC method are shown in the left panel with the band representations near $\Gamma$ point indicated in the inset. Calculations in the paramagnetic state using the $\mathrm{mBJ}$ method with $c_{\mathrm{MBJ}}=1.18$ are shown in the right panel. The band inversion disappears and a trivial band gap of about $10 \mathrm{meV}$ opens along the $Y-\Gamma$ direction.

\section{Band structure calculations}

To shed light on the possible topological nature of the band structure of $\mathrm{Eu}_{5} \mathrm{In}_{2} \mathrm{Sb}_{6}$, we perform band structure calculations in the paramagnetic state by taking the $4 f$ orbitals of Eu as core states, as shown in Fig. 5. Both barium and europium are divalent in the 526 structure, and our experimental results imply that europium has a well-localized $f$-electron contribution. One would therefore naively expect that the band structure and topology of $E u_{5} I_{2} S_{6}$ are similar to that of $B_{5} I_{2} \mathrm{n}_{2} \mathrm{Sb}_{6}$, whose topology is not indicated by any symmetry indicators but can be characterized by nontrivial connecting pattern in the Wilson bands ${ }^{27}$.

Remarkably, GGA + SOC calculations in the paramagnetic state of $\mathrm{Eu}_{5} \mathrm{In}_{2} \mathrm{Sb}_{6}$ indicate a semimetal state with one extra band inversion compared to $\mathrm{Ba}_{5} \mathrm{In}_{2} \mathrm{Sb}_{6}$ at the $\Gamma$ point. Because there are no symmetry-protected band crossings between the valence and conduction bands at any $k$-point, a $k$-dependent chemical potential can be defined, which yields a fully gapped state. By calculating the topological indices of the bands below the $k$ dependent chemical potential, we find that the extra band inversion at $\Gamma$ point yields a strong topological insulator with $\left(z_{2}\right.$; $\left.z_{2 w, 1} z_{2 w 2} z_{2 w, 3}\right)=(1 ; 000)$, where $z_{2}$ is strong index and $z_{2 w, i}$ is weak index $^{43}$, as shown in Fig. 5a. Compared with our experimental results, however, the ab initio calculation with the GGA functional incorrectly predicts $\mathrm{Eu}_{5} \mathrm{In}_{2} \mathrm{Sb}_{6}$ to be semimetallic. Considering the possible underestimation of the band gap in semiconductors by the GGA functional, we have also performed band structure calculations using the $\mathrm{mBJ}$ potential with a coefficient $c_{\mathrm{MBJ}}=1.18$, which was obtained self-consistently. As shown in Fig. 5b, the band inversion near the $\Gamma$ point disappears, and a small gap opens along the $\Gamma-Y$ path. The topological indices $\left(z_{2} ; z_{2 w, 1} z_{2 w 2} z_{2 w, 3}\right)$ are computed to be $(0 ; 000)$. In fact, surface states are not detected by our electrical transport measurements. Scanning tunneling microscopy and angle-resolved photoemission measurements will be valuable to confirm the absence of in-gap states.

We now investigate the topology of $\mathrm{Eu}_{5} \mathrm{In}_{2} \mathrm{Sb}_{6}$ in the magnetically ordered state. Because the magnetic structure of $\mathrm{Eu}_{5} \mathrm{In}_{2} \mathrm{Sb}_{6}$ has not been solved yet, we investigate theoretically, using the GGA $+U+$ SOC approach, three A-type AFM phases with the easy axis along different directions. All of the antiferromagnetic phases are theoretically characterized by the so-called Type-IV magnetic space groups (MSGs) with inversion symmetry. The magnetic topological quantum chemistry theory therefore describes the topology of these MSGs by an index group $\left(Z_{4} \times Z_{2}^{3}\right)$, as proposed recently ${ }^{44}$. From the calculations detailed in Supplementary Fig. 5 , the magnetic moment is about $7 \mu_{B} / \mathrm{Eu}$, and the energy difference between the different phases is within
$3 \mathrm{meV}$ per unit cell. From the results tabulated in Supplementary Table 1, all three AFM phases are axion insulators with strong indices $\left(z_{4}, z_{2_{1}}, z_{2_{2}}, z_{2_{3}}\right)=(2,0,0,0)$. By comparing the band structures for three different AFM phases, the polarized $4 f$ states do not change the band inversion characteristics of the paramagnetic state but induce a small exchange splitting near the Fermi level. Though the AFM structure at low temperatures has yet to be determined experimentally, we proposed that this phase is an axion insulator candidate that preserves inversion symmetry.

\section{DISCUSSION}

The magnetic polaron picture is fully consistent with our data. At high temperatures $\left(\sim 15 T_{\mathrm{N} 1}=210 \mathrm{~K}\right)$, the formation of isolated magnetic polarons is manifested in magnetic susceptibility measurements via a deviation from the Curie-Weiss law (inset of Fig. 1a) and in electrical resistivity measurements via the onset of negative magnetoresistance (Fig. 2c). As the temperature is further lowered, these polarons increase in size until they start to interact at $T^{*}$ giving rise to a sharp decrease in the $X^{T}$ plot, a Schottky anomaly in the specific heat data (Fig. 1c), and an anomaly in electrical resistivity measurements (Fig. 2b). At $T_{N 1}$, the polarons coalesce and become delocalized, which gives way to a drastic increase in conductivity. Though the delocalization temperature virtually coincides with $T_{\mathrm{N} 1}$ at zero field, delocalization is expected to occur at higher temperatures as the size of the polarons increase in field. Antiferromagnetic-driven $T^{*}$, however, is suppressed in field. This opposite field dependence causes the delocalization temperature and $T^{*}$ to merge into one at about $3 \mathrm{~T}$, which gives rise to a resistivity maximum above $T_{\mathrm{N} 1}$ that moves to higher temperatures in field (see Supplementary Fig. 7). Importantly, the increase in size of magnetic polarons in applied fields also promotes large negative (termed colossal) magnetoresistance in the paramagnetic state. In fact, CMR sets in at about $200 \mathrm{~K}$ and peaks just above $T_{\mathrm{N} 1}$, as shown in Fig. $2 \mathrm{C}$.

Another characteristic of CMR materials is the scaling of the lowfield $M R$ with the square of the reduced magnetization, $\Delta \rho / \rho_{0}=C\left(M / M_{\text {sat }}\right)^{2}$, where $M_{\text {sat }}$ is the saturation magnetization $^{3,45}$. Just above $T_{\mathrm{N} 1}$, this scaling is valid and yields $C=50$ (inset of Fig. 2c). When electron scattering is dominated by magnetic fluctuations, the scaling constant $C$ is proportional to $n^{-2 / 3}, n$ being the carrier density ${ }^{36}$. The scaling constant calculated this way $\left(n \sim 10^{12} / \mathrm{cm}^{3}\right.$ at $\left.15 \mathrm{~K}\right)$ is four orders of magnitude higher than the experimentally determined constant, which is an indication of a distinct mechanism. Another notable exception is $\mathrm{EuB}_{6}$, for which the field-dependent resistivity was argued to be 
dominated by the increase in polaron size with field rather than by the suppression of critical scattering ${ }^{17,46}$. In fact, recent scanning tunneling microscopy measurements have directly imaged the formation of magnetic polarons in $\mathrm{EuB}_{6}{ }^{20}$.

In summary, we investigate the thermodynamic and electrical transport properties of single crystalline $\mathrm{Eu}_{5} \mathrm{In}_{2} \mathrm{Sb}_{6}$, a nonsymmorphic Zintl antiferromagnetic insulator. Colossal magnetoresistance sets in at temperatures one order of magnitude higher than the magnetic ordering temperature, $T_{\mathrm{N} 1}=14 \mathrm{~K}$, and peaks just above $T_{\mathrm{N} 1}$ reaching $-99.7 \%$ at $3 \mathrm{~T}$ and $-99.999 \%$ at $9 \mathrm{~T}$. This is, to our knowledge, the largest CMR observed in a stoichiometric antiferromagnetic compound. Our combined electrical transport and microscopic ESR measurements point to the presence of magnetic polarons that generate an anomalous Hall component. Our first-principles band structure calculations yield an insulating state with trivial topological indices in the paramagnetic state, whereas an axion insulating state emerges within putative antiferromagnetic states. Our results highlight that Zintl phases could provide truly insulating states in the search for topological insulators, and rare-earth elements provide a route for the discovery of topological interacting phenomena. In fact, Zintl $\mathrm{EuX}_{2} \mathrm{As}_{2}(X=\mathrm{ln}, \mathrm{Sn})$ have been recently proposed to be antiferromagnetic topological insulators ${ }^{47,48}$. The metallic-like behavior observed in electrical resistivity, however, suggests that these materials have a semimetallic ground state akin to $\mathrm{EuB}_{6}{ }^{49}$.

\section{METHODS}

Experimental details

Single crystalline samples of $\mathrm{Eu}_{5} \mathrm{In}_{2} \mathrm{Sb}_{6}$ were grown using a combined $\mathrm{In}$-Sb self-flux technique. The crystallographic structure was verified at room temperature by both single-crystal diffraction using Mo radiation in a commercial diffractometer (see Supplementary Fig. 6) and powder diffraction using $\mathrm{Cu}$ radiation in a commercial diffractometer. $\mathrm{Eu}_{5} \mathrm{In}_{2} \mathrm{Sb}_{6}$ crystallizes in an orthorhombic structure (space group 55) with lattice parameters $a=12.553(5) \AA, b=14.603(2) \AA$ and $c=4.635(1) \AA$. As shown in Supplementary Fig. 6 , the observed mosaicity of the Bragg reflections is limited by the resolution of the diffractometer. The crystals have a rod-like shape, the $c$-axis is the long axis, and typical sizes are $0.5 \mathrm{~mm} \times 0.5 \mathrm{~mm} \times$ $3 \mathrm{~mm}$. In addition, the stoichiometry of crystals was checked by energy dispersive X-ray spectroscopy (EDX). Magnetization measurements were performed in a commercial SQUID-based magnetometer. Specific heat measurements were made using the thermal relaxation technique in a commercial measurement system. Because of the difficulties in the synthesis of phase pure $\mathrm{Ba}_{5} \mathrm{In}_{2} \mathrm{Sb}_{6}$, no phonon background was subtracted from the data. A four-probe configuration was used in the electrical resistivity experiments performed using a low-frequency AC bridge. Highfield magnetization measurements were performed in the $65 \mathrm{~T}$ pulse field magnet at $4 \mathrm{~K}$ at the National High Magnetic Field Laboratory at Los Alamos National Laboratory. Details of the magnetometer design are described in ref. ${ }^{50}$. The sample was mounted in a plastic cup oriented with $b$-axis parallel to the magnetic field. The data were normalized by the lowfield data obtained from a commercial SQUID magnetometer.

ESR measurements were performed on single crystals in $X$-band $(f=$ $9.5 \mathrm{GHz})$ and $Q$-band $(f=34 \mathrm{GHz})$ spectrometers equipped with a goniometer and a He-flow cryostat in the temperature range of $4 \mathrm{~K}<T<300 \mathrm{~K}$.

\section{Theoretical details}

First-principle calculations were performed using the Vienna ab initio simulation package (VASP), and the GGA with the Perdew-Burke -Ernzerhof (PBE) type exchange correlation potential was adopted. The Brillouin zone (BZ) sampling was performed by using $k$ grids with an $7 \times$ $7 \times 9$ mesh in self-consistent calculations. In the paramagnetic state, we employed a europium pseudopotential with seven $f$ electrons treated as core electrons. In the antiferromagnetic states, we performed the LSDA + $U$ calculations with $U=5 \mathrm{eV}$ for the three distinct magnetic structures.

\section{DATA AVAILABILITY}

Data presented in this study are available from authors upon request.
Received: 21 October 2019; Accepted: 6 July 2020; Published online: 24 July 2020

\section{REFERENCES}

1. Tomczak, J. M. Thermoelectricity in correlated narrow-gap semiconductors. J. Phys. Condens. Matter 30, 183001 (2018).

2. Hochberg, Y. et al. Detection of sub-MeV dark matter with three-dimensional Dirac materials. Phys. Rev. D 97, 015004 (2018).

3. Hasan, M. Z. \& Kane, C. L. Colloquium: Topological insulators. Rev. Mod. Phys. 82 , 3045-3067 (2010).

4. Moore, J. E. The birth of topological insulators. Nature 464, 194-198 (2010).

5. Hasan, M. Z. \& Moore, J. E. Three-dimensional topological insulators. Annu. Rev. Condens. Matter Phys. 2, 55-78 (2011).

6. Cava, R. J., Ji, H., Fuccillo, M. K., Gibson, Q. D. \& Hor, Y. S. Crystal structure and chemistry of topological insulators. J. Mater. Chem. C 1, 3176-3189 (2013).

7. Eo, Y. S. et al. Transport gap in $\mathrm{SmB}_{6}$ protected against disorder. Proc. Natl. Acad. Sci. USA 116, 12638-12641 (2019).

8. Sato, Y. et al. Unconventional thermal metallic state of charge-neutral fermions in an insulator. Nat. Phys. 15, 954-959 (2019).

9. Sun, Y. et al. Strain-driven onset of nontrivial topological insulating states in Zintl $\mathrm{Sr}_{2} X$ compounds $(X=\mathrm{Pb}, \mathrm{Sn})$. Phys. Rev. B 84, 165127 (2011).

10. Wang, L.-L., Kaminski, A., Canfield, P. C. \& Johnson, D. D. Different topological quantum states in ternary Zintl compounds: $\mathrm{BaCa} X(X=\mathrm{Si}, \mathrm{Ge}, \mathrm{Sn}$ and $\mathrm{Pb})$. J. Phys. Chem. C 122, 705-713 (2018).

11. Chang, C.-Z. Marriage of topology and magnetism. Nat. Mater. 19, 484-485 (2020).

12. Yu, R. et al. Quantized anomalous Hall effect in magnetic topological insulators. Science 329, 61-64 (2010).

13. Zhang, D. et al. Topological axion states in the magnetic insulator $\mathrm{MnBi}_{2} \mathrm{Te}_{4}$ with the quantized magnetoelectric effect. Phys. Rev. Lett. 122, 206401 (2019).

14. Shapira, Y., Foner, S., Oliveira, N. F. \& Reed, T. B. EuTe. II. Resistivity and Hall effect. Phys. Rev. B 5, 2647-2657 (1972).

15. Chan, J. Y., Kauzlarich, S. M., Klavins, P., Shelton, R. N. \& Webb, D. J. Colossal magnetoresistance in the transition-metal Zintl compound $\mathrm{Eu}_{14} \mathrm{MnSb}_{11}$. Chem. Mater. 9, 3132-3135 (1997).

16. Chan, J. Y., Kauzlarich, S. M., Klavins, P., Shelton, R. N. \& Webb, D. J. Colossal negative magnetoresistance in an antiferromagnet. Phys. Rev. B 57, R8103-R8106 (1998).

17. Süllow, S. et al. Magnetotransport in the low carrier density ferromagnet EuB $6 . J$. Appl. Phys. 87, 5591-5593 (2000).

18. Devlin, K. P. et al. Eu $\mathrm{uu}_{11} \mathrm{Zn}_{4} \mathrm{Sn}_{2} \mathrm{As}_{12}$ : a ferromagnetic Zintl semiconductor with a layered structure featuring extended $\mathrm{Zn}_{4} \mathrm{As}_{6}$ Sheets and ethane-like $\mathrm{Sn}_{2} \mathrm{As}_{6}$ units. Chem. Mater. 30, 7067-7076 (2018).

19. Kasuya, T. \& Yanase, A. Anomalous transport phenomena in Eu-chalcogenide alloys. Rev. Mod. Phys. 40, 684-696 (1968).

20. Pohlit, M. et al. Evidence for ferromagnetic clusters in the colossalmagnetoresistance material EuB 6 . Phys. Rev. Lett. 120, 257201 (2018).

21. Jin, S. et al. Thousandfold change in resistivity in magnetoresistive La-Ca-Mn-O films. Science 264, 413-415 (1994).

22. Salamon, M. B. \& Jaime, M. The physics of manganites: structure and transport. Rev. Mod. Phys. 73, 583-628 (2001).

23. Sakurai, $\mathrm{H}$. Novel colossal magnetoresistance in $\mathrm{NaCr}_{2} \mathrm{O}_{4}$. J. Kor. Phys. Soc. 63 , 583-586 (2013).

24. Baltz, V. et al. Antiferromagnetic spintronics. Rev. Mod. Phys. 90, 015005 (2018).

25. Young, S. M. \& Kane, C. L. Dirac semimetals in two dimensions. Phys. Rev. Lett. 115, 126803 (2015).

26. Parameswaran, S. A., Turner, A. M., Arovas, D. P. \& Vishwanath, A. Topological order and absence of band insulators at integer filling in non-symmorphic crystals. Nat. Phys. 9, 299-303 (2013).

27. Wieder, B. J. et al. Wallpaper fermions and the nonsymmorphic Dirac insulator Science 361, 246-251 (2018).

28. Bradlyn, B. et al. Topological quantum chemistry. Nature 547, 198-305 (2017).

29. Vergniory, M. G. et al. A complete catalogue of high-quality topological materials. Nature 566, 480-485 (2019).

30. Zhang, T. et al. Catalogue of topological electronic materials. Nature 566, 475-479 (2019).

31. Tang, F., Po, H. C., Vishwanath, A. \& Wan, X. Comprehensive search for topological materials using symmetry indicators. Nature 566, 486-489 (2019).

32. Subbarao, U., Sarkar, S., Joseph, B. \& Peter, S. C. Magnetic and X-ray absorption studies on the $R E_{5} X_{2} \mathrm{Sb}_{6}(R E=\mathrm{Eu}, \mathrm{Yb} ; X=\mathrm{Al}, \mathrm{Ga}, \mathrm{In})$ compounds. J. Alloy. Compd. 658, 395-401 (2016).

33. Ruck, B. J. et al. Magnetic state of EuN: X-ray magnetic circular dichroism at the Eu $M_{4,5}$ and $L_{2,3}$ absorption edges. Phys. Rev. B 83, 174404 (2011). 
34. Radzieowski, M. et al. On the divalent character of the Eu atoms in the ternary Zintl phases $\mathrm{Eu}_{5} \mathrm{In}_{2} P \mathrm{n}_{6}$ and $\mathrm{Eu}_{3} \mathrm{MAs}_{3}(P n=\mathrm{As}-\mathrm{Bi} ; M=\mathrm{Al}, \mathrm{Ga})$. Mater. Chem. Front. 4, 1231-1248 (2020).

35. Teresa, J. M. D. et al. Evidence for magnetic polarons in the magnetoresistive perovskites. Nature 386, 256-259 (1997).

36. Majumdar, P. \& Littlewood, P. Magnetoresistance in Mn pyrochlore: electrical transport in a low carrier density ferromagnet. Phys. Rev. Lett. 81, 1314-1317 (1998).

37. Cordier, G. \& Stelter, M. $\mathrm{Sr}_{5} \mathrm{Al}_{2} \mathrm{Sb}_{6}$ and $\mathrm{Ba}_{5} \mathrm{In}_{2} \mathrm{Sb}_{6}$ : two new Zintl phases with different chain anions. Z. Naturforsch. B 43, 463-466 (1988).

38. Emin, D. \& Holstein, T. Studies of small-polaron motion IV. Adiabatic theory of the Hall effect. Ann. Phys. 53, 439-520 (1969).

39. Urbano, R. R. et al. Magnetic polaron and Fermi surface effects in the spin-flip scattering of $\mathrm{EuB}_{6}$. Phys. Rev. B 70, 140401 (2004).

40. Rosa, P. F. S. et al. Electron spin resonance of the intermetallic antiferromagnet Euln ${ }_{2} \mathrm{As}_{2}$. Phys. Rev. B 86, 094408 (2012).

41. Huber, D. L. Analysis of electron paramagnetic resonance experiments in colossal magnetoresistance materials. J. Appl. Phys. 83, 6949-6951 (1998).

42. Yang, Z., Bao, X., Tan, S. \& Zhang, Y. Magnetic polaron conduction in the colossal magnetoresistance material $\mathrm{Fe}_{1-x} \mathrm{Cd}_{x} \mathrm{Cr}_{2} \mathrm{~S}_{4}$. Phys. Rev. B 69, 144407 (2004).

43. Fu, L. \& Kane, C. L. Topological insulators with inversion symmetry. Phys. Rev. B 76, 045302 (2007)

44. $\mathrm{Xu}, \mathrm{Y}$. et al. High-throughput calculations of antiferromagnetic topological materials from magnetic topological quantum chemistry. Preprint at https://arxiv. org/abs/2003.00012 (2020).

45. Furukawa, N. Transport properties of the Kondo lattice model in the limit $S=\infty$ and $D=\infty$. J. Phys. Soc. Jpn. 63, 3214-3217 (1994).

46. Süllow, S. et al. Metallization and magnetic order in EuB 6 . Phys. Rev. B 62, 11626-11632 (2000).

47. Xu, Y., Song, Z., Wang, Z., Weng, H. \& Dai, X. Higher-order topology of the axion insulator Euln ${ }_{2} \mathrm{As}_{2}$. Phys. Rev. Lett. 122, 256402 (2019).

48. $\mathrm{Li}, \mathrm{H}$. et al. Dirac surface states in intrinsic magnetic topological insulators EuSn $\mathrm{As}_{2}$ and $\mathrm{MnBi}_{2 n} \mathrm{Te}_{3 n+1}$. Phys. Rev. X 9, 041039 (2019).

49. Nie, S. et al. Magnetic semimetals and quantized anomalous Hall effect in $\mathrm{EuB}_{6}$. Phys. Rev. Lett. 124, 076403 (2020).

50. Detwiler, J. A. et al. Magnetization of UBe ${ }_{13}$ to 60 T. Phys. Rev. B 61, 402-404 (2000).

\section{ACKNOWLEDGEMENTS}

We acknowledge constructive discussions with Z. Fisk, S. Wirth, J. Muller, O. Erten and C. Kurdak. Synthesis and macroscopic measurements at low fields were supported by the U.S. Department of Energy (DOE) BES "Quantum Fluctuations in Narrow-Band Systems" project. High-field magnetization measurements were supported by the DOE BES "Science of 100 Tesla" project. S.K. acknowledges support from the LANL Director's Postdoctoral LDRD program. The National High Magnetic Field Laboratory is supported by the National Science Foundation through NSF/DMR-1644779 and the State of Florida. Scanning electron microscope and energy dispersive X-ray measurements were performed at the Center for Integrated Nanotechnologies, an Office of Science User Facility operated for the DOE Office of Science. Electron spin resonance measurements were supported by FAPESP (SP-Brazil) grants no. 2018/ 11364-7, 2017/10581-1, 2012/04870-7, CNPq Grant no. 141026/2017-0, CAPES and FINEP-Brazil. We thank Diamond Light Source for the provision of beamtime under proposal MT18991. Work at UCL is supported by the UK Engineering and Physical
Sciences Research Council (Grants No. EP/N027671/1 and No. EP/N034694/1) Theory efforts were supported by DOE de-sc0016239, NSF EAGER 1004957, Simons Investigator Grants, ARO MURI W911NF- 12-1-0461, the Packard Foundation, and the Schmidt Fund for Innovative Research. Y.X. and A. Bernevig were supported by Max Planck society. Z.W. received support from the National Natural Science Foundation of China (No. 11974395), the Chinese Academy of Sciences (CAS) (Grant No. XDB33000000), and the CAS Pioneer Hundred Talents Program.

\section{AUTHOR CONTRIBUTIONS}

P.R. and F.R. conceived the project. P.R. and E.B. synthesized single crystals for the measurement. P.R. and S.T. performed electrical transport and specific heat measurements. J.T. performed magnetization measurements to 6T. S.K., M.C., and N.H. performed magnetization measurements to $60 \mathrm{~T}$. J.S. and P.P. performed electron spin resonance measurements. M.R., L.V., M.J., and A. Bombardi performed Xray absorption measurements measurements. Y.X., Z.W., and A. Bernevig performed band structure calculations. P.R. wrote the manuscript with input from all authors.

\section{COMPETING INTERESTS}

The authors declare no competing interests.

\section{ADDITIONAL INFORMATION}

Supplementary information is available for this paper at https://doi.org/10.1038/ s41535-020-00256-8.

Correspondence and requests for materials should be addressed to P.R.

Reprints and permission information is available at http://www.nature.com/ reprints

Publisher's note Springer Nature remains neutral with regard to jurisdictional claims in published maps and institutional affiliations.

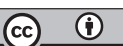

Open Access This article is licensed under a Creative Commons Attribution 4.0 International License, which permits use, sharing, adaptation, distribution and reproduction in any medium or format, as long as you give appropriate credit to the original author(s) and the source, provide a link to the Creative Commons license, and indicate if changes were made. The images or other third party material in this article are included in the article's Creative Commons license, unless indicated otherwise in a credit line to the material. If material is not included in the article's Creative Commons license and your intended use is not permitted by statutory regulation or exceeds the permitted use, you will need to obtain permission directly from the copyright holder. To view a copy of this license, visit http://creativecommons. org/licenses/by/4.0/.

(c) The Author(s) 2020 